\title{
Brain response to focal vibro-tactile stimulation prior to muscle contraction
}

\author{
Tijana Jevtic, Aleksandar Zivanovic \\ School of Science and Technology, Middlesex University, \\ The Burroughs, London, NW4 4BT, UK \\ \{t.jevtic; a.zivanovic\}@mdx.ac.uk
}

\author{
Rui C.V. Loureiro \\ Aspire Centre for Rehabilitation Engineering and Assistive \\ Technology, University College London, Royal National \\ Orthopaedic Hospital, Stanmore, London, HA7 4LP, UK \\ r.loureiro@ucl.ac.uk
}

\begin{abstract}
This paper presents a single case study of an ongoing study evaluating cortical association with facilitation and management of vibro-tactile stimulation applied prior to voluntary muscle contraction. The study consisted of three repetitions of relaxation phase during which vibrations are applied; and a contraction phase. EEG and EMG data was collected to determine muscle and brain activation patterns. The EEG analysis of the mu waves during relaxation + vibration phase seem to indicate sensory cortex activation during focal muscle vibrations. With repetitiveness of vibrations, an increase in maximal calculated mu power was observed that could suggest optimization of the muscle fibers prior to the contraction. When contraction is performed, mu waves are desynchronizing with the movement execution. The analysis of the last relaxation period indicate that the muscle itself facilitates the last contraction locally possibly due to cortical learning
\end{abstract}

Keywords-muscle performance; surface electroenchephalography; EEG; EMG; vibro-tactile stimulation; robot-mediated neurorehabilitation

\section{INTRODUCTION}

Combining robotic technology with physiotherapy exercises could contribute to improved rehabilitation outcomes. One of the main goals of intelligent technology driven environments is to develop affordable, portable devices easy to use in unsupervised environments such as the home. This availability can have an impact on patients' motivation and increased training at the patient's convenience as it is shown to have beneficial rehabilitation outcomes [1]. Current technological trends provide an opportunity to combine kinaesthetic feedback with tactile stimulation for preconditioning muscles and take advantage of the robotic therapy paradigms in achieving peripheral plasticity and functional recovery [2].

There is a growing interest in using vibro-tactile stimulation in rehabilitation. The greatest body of work has been conducted with a whole body vibration (WBV). However the size and cost of WBV devices limit it to specified and supervised use. Therefore a focus should shift to more usable vibration devices such as smaller coin shaped motors that can target specific muscle groups by providing segmental or focal vibro-tactile stimulation [3], [4].
The literature provides uprising evidence of the impact of focal vibro-tactile stimulation on healthy and impaired people [5], [6]. Focal vibrations (FV) are being used in sport science to enhance muscle output and extend exercise performance. Nevertheless FV are used to reduce abnormally increased muscle tone (reflected in spasticity) or to use as a proprioceptive training method to restore sensorimotor organization in movement disorders [7].

Although vibro-tactile rehabilitation paradigms have been widely used, the reason as to why such vibrations are effective and the underlying mechanisms perceiving/ facilitating vibrotactile stimulation response remains unclear. It has been postulated that the mechanism relates to type I and II afferent activation and spinal stretch reflex response to the stimuli [8]. However this claim cannot be applied to people with a spinal cord injury (SCI), i.e. when vibrations are applied below the level of injury to treat spasticity [9]. If the spine is responsible for a muscle response to vibrations, it is unclear why high muscle tone reduces when the communication with the central nervous system via the spinal cord is compromised.

Thus the question remains as to how the nervous system responds to vibration and how to best optimise therapy based on vibro-tactile stimulation. Is the response to a vibro-tactile stimulation local (e.g. at muscle level), spinal (e.g. spinal reflexes) or a CNS response (from sensorimotor cortex)? Or is it a combination of all three?

In this paper a single case study examining brain response to focal vibro-tactile stimulation of a muscle is presented. The study focuses on the analysis of mu activity over the sensorimotor cortex to evaluate how the brain perceives vibrotactile stimulation.

\section{BACKGROUND}

Signals from the brain can be recorded using electroencephalography (EEG). Signals recorded from the brain are categorised according to the brain activity such as relaxation, movement execution, eye blinking, external stimulation, etc. Alpha waves (range $7-15 \mathrm{~Hz}$ ) are observed over the parietal part of the brain during relaxation. Beta waves (range 16-32Hz) from the motor and premotor cortex 
correspond to planning and executing the movement. Delta waves (range $0.5-4 \mathrm{~Hz}$ ) are associated with deep sleep.

$\mathrm{Mu}$ or $\mu$ waves (range $8-12 \mathrm{~Hz}$ ) are observed over sensorimotor cortex and are associated with muscle and joint perception and motion coordination. Increase in mu rhythm can be noticed when the body is relaxed with no intention for movement. $\mathrm{Mu}$ desynchronization, i.e. decrease in amplitude, occurs when the movement is planned and executed. Some research correlate mu desynchronization even when the person is consciously observing someone else's movements [10].

Because mu waves are correlated with muscle activity, this range of waves is selected for the analysis in this study. As this study design corresponds to alterations of relaxation and contraction periods, with vibrations being applied during relaxation, the mu waves' analysis could potentially contribute to better understanding of cortical acumen of focal muscle vibrations.

\section{Metodology}

\section{A. Subject}

This paper presents a case study of an on-going larger study. The participant was left-handed healthy male volunteer. The experiments were performed with approval of the local Ethics Committee. The participant gave informed consent to the experimental procedure as required by the Helsinki declaration (1964).

\section{B. Experimental Setup}

The experimental setup is adapted from previous study [3]. The participant was asked to rest the hand on a table, in a midsuppination position. At specified times during the study participant were instructed to abduct the index finger by pushing against the force gauge positioned above the distal phalange to achieve muscle contraction or to rest. The vibrations are applied over a muscle belly of the first interosseous muscle during the relaxation period (i.e. before the contraction). Experimental protocol comprised of three repetition of relaxation period lasting 60 seconds followed by 20 seconds of muscle contraction. The times were chosen in such a way to provide enough time to record muscle's responses and for a muscle to recover from a contraction, while three repetitions were chosen to ensure statistical coherency while limiting time bourdon on the participant. During contraction phase participant were asked to maintain the specified force limits, which represented $80 \% \pm 5 \%$ of maximal voluntary contraction force level.

Focal vibrations are applied using a small vibration motor (12mm Pico Vibe ${ }^{\mathrm{TM}}$ ) attached to an Arduino microcontroller. The frequency of the vibrations generated by the motor was $30 \mathrm{~Hz}$ as this frequency is find to be the most fatiguing [5].

EEG was recorded using TMSi water based electrodes connected to a porti7 amplifier (TMSi, Netherlands). Electrode placements were corresponding to the TMSi water adapted 1020 system cap presented in Fig. 1.

\section{Data Analysis}

The signal processing and analysis were conducted in MATLAB ${ }^{\circledR}$ using well-established functions and signal processing toolboxes. The analysis was performed with the whole signal from every electrode and additionally on cut portions of the signals. Each signal was time stamped and cut corresponding to the experimental phase i.e. relaxation or contraction.

The raw signals were band pass filtered between 5 and $35 \mathrm{~Hz}$ using two-way least-squares FIR filtering. Time frequency domain was represented using a spectrogram function, which calculates time-frequency dependency based on short time Fourier transformation. Power spectral density was calculated using the Welch method on both whole signal and on cut subsignals.

After the Welch power spectrum was calculated for subsignals, the maximum value of mu band was extracted (8$12 \mathrm{~Hz}$ ). The maximum(s) were represented with a topographic heatmap function corresponding the electrode placement on a head.
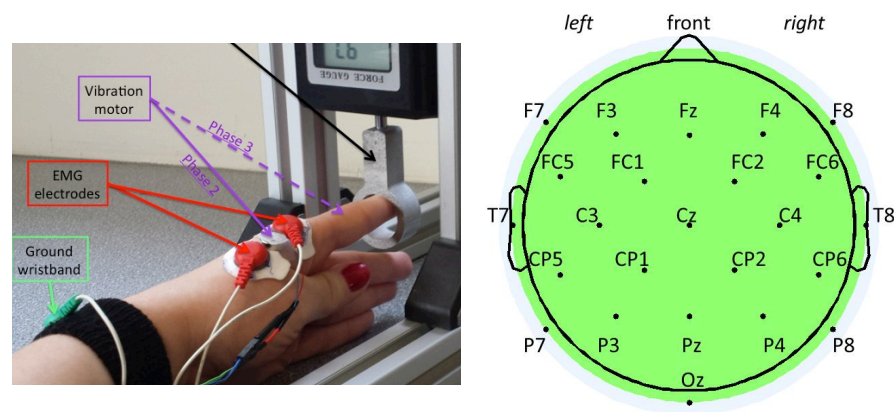

Fig. 1. Experimental setup (left) and EEG electrode placement (10-20 system) (Right).

\section{RESULTS}

The spectrogram of the CP2 electrode, corresponding to the sensory cortex, is presented in Fig. 2. The power spectral density using the Welch method of the CP2 electrode is presented in Fig. 3. On a single topographic head hat map all electrodes are represented corresponding to their spatial placement over the brain (any topograph in Fig. 4). Each electrode is presented with corresponding maximal Welch power spectral density of the mu wave. Six topographs are lined up based on the time of phase execution during the experiment (i.e. vibration condition) in Fig. 4. Force and amplitude of EMG can be seen in Fig. 5. 


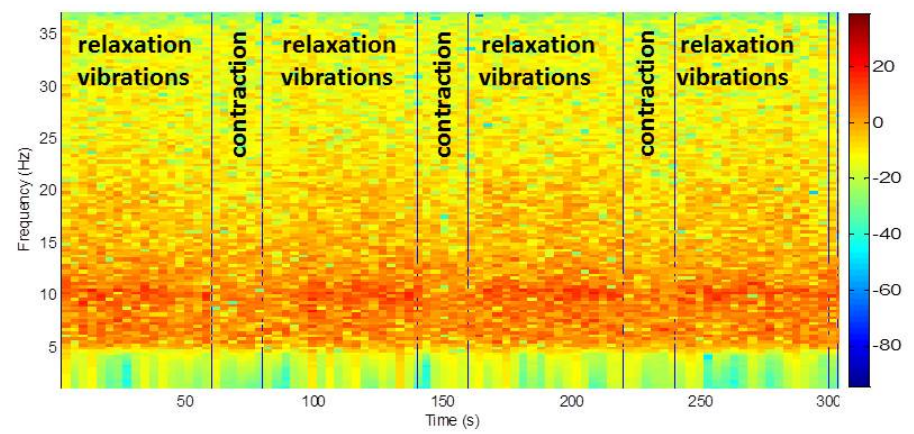

Fig. 2. Spectrogram of the CP2 electrode. Vertical lines separate the different phases during the experimental protocol: three repetitions of relaxation followed by contraction.

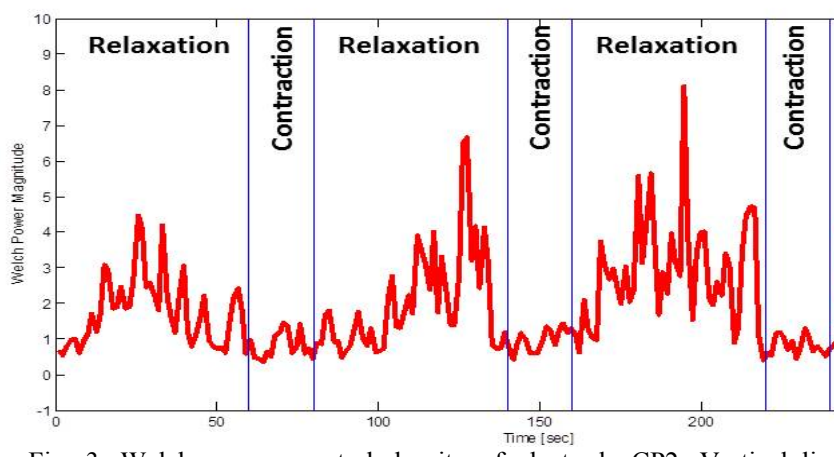

Fig. 3. Welch power spectral density of electrode CP2. Vertical lines separate the different phases during experimental protocol: three repetitions of relaxation each followed by consecutive contraction, conducted as one sequence.

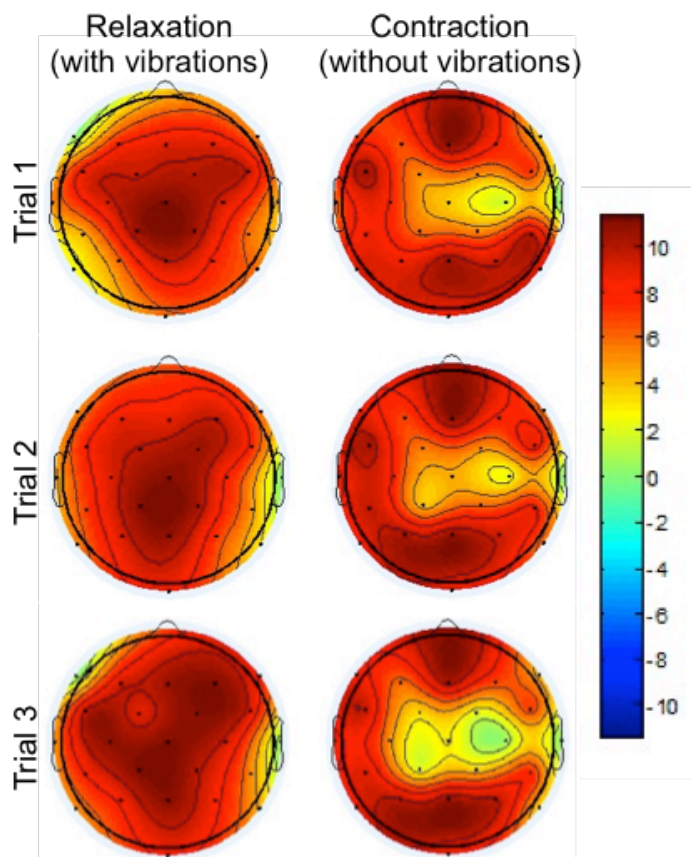

Fig. 4. Topographic head heatmaps corresponding physical placements of all of the electrodes. The results are presented for each consecutive trial during the study: relaxation and contraction.
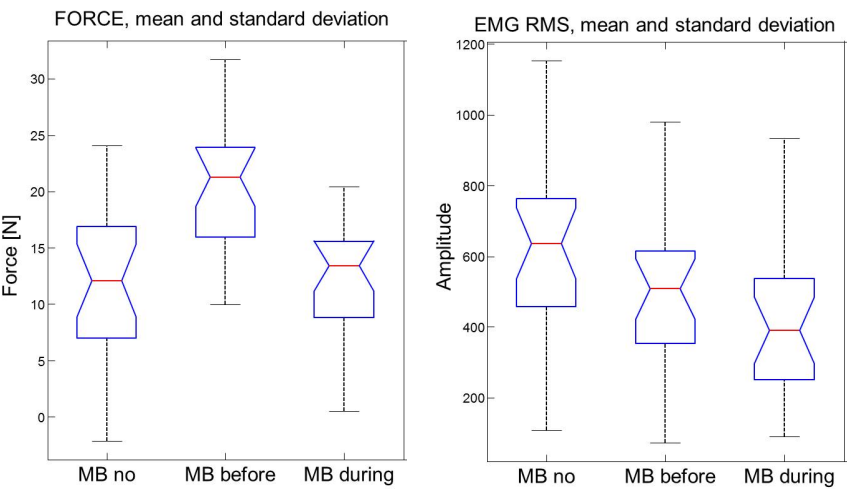

Fig. 5. Force (left) exerted by the participant and amplitude of EMG (right) when the focal vibrations are applied on the muscle belly (MB). The 10-40\% increase in force and the 10-20\% decrease in EMG amplitude is noticed when vibrations are applied during the relaxation period (second bars on both figures).

\section{DISCUSSION}

Visual inspection of the spectrogram shown on Fig. 2, seems to indicate an increase in mu power when vibrations are applied during relaxation period. This might relate to coactivation of the sensorimotor cortex during the relaxation period due to the vibro-tactile stimuli. Therefore it looks like the brain also perceives the external stimuli and perhaps vibrotactile perception it's not only localized to muscles and spine [11].

Due to spectrogram limitations and poor resolution, further analysis was conducted in order to obtain Welch power spectral density. Welch's power spectral density shown in Fig. 3 represents the mu power envelope throughout the duration of the experiment. The increase in $\mathrm{mu}$ power can be observed during the relaxation period with vibrations. The drop in the mu power during contraction might be mu desynchronization corresponding to the movement execution. In line with Fig. 3, the highest magnitude of mu power over the last relaxation period is present in all electrodes of Welch's power spectrum. This high magnitude over the last relaxation period could possibly suggest that vibrations enhance muscle memory recall in conjunction with motor learning [12]. Vibro-tactile preconditioning in this context would tap into muscle memory in order to optimize upcoming contraction in parallel with repetition-based learning. The repetition would thus facilitate the brain to potentially predetermine, optimise and execute the following contraction [13] of the vibrated muscle, i.e. one would argue towards the possibility of vibration-evoked neuroplasticity.

Fig. 4 shows an increase in mu waves over the sensory cortex during the relaxation with vibrations phase. This corresponds to the nature of the mu waves during relaxation. Conversely, as the mu waves can also associate with the muscle activation, consistency with the decreased peak values over the $\mathrm{CP} 1, \mathrm{Cz}$ and CP2 electrodes suggest the feasibility of detecting mu waves as a response to the focal muscle vibrations. On the other hand, de-synchronization of the mu waves can be observed during the contraction phase over the central sensorimotor cortex that correlates to movement execution. 
Interestingly both of the effects are pronounced during the last, third relaxation and contraction phases. Although we cannot make any assumptions or conclusions from one single case study, the results seem to suggest that perhaps the brain learned how to respond to the repetitive vibro-tactile stimuli, noting that the stimulation and the contraction parameters and requirements are not changing during the time. And this assumption is in line with the proposed analysis of the Welch power density shown in Fig. 3.

Further analysis of the mu waves spectral density peaks over the motor cortex during relaxation + vibration phase might be suggesting cortical reorganization due to the upcoming movement planning. Because it is the third consecutive repetition of same tasks, could the muscles memorize the optimum activation without muscle stabilization? Although more evidence is needed and based on a single case study one cannot drawn any conclusions, the assumption above seems to indicate that the brain has no involvement in the upcoming contraction because the muscle itself can manage it locally.

Vibration induced neuroplasticity and cerebral-muscular shared control reserves a deeper discussion. During the first relaxation phase, it is assumed that the brain perceives foreign vibro-tactile stimuli and responds to it with the intention to stabilize the muscle. The stabilization could potentially be achieved with increased muscle stretch and possibly increase in muscle spindles' and Golgi tendon organs' type I and II afferent pathways thresholds [14]. Because the now potentially stretched muscle has greater leverage, the brain responds with a lower number of active muscle units to perform the task. These assumptions can be further exercised in Fig. 5 where the increase in force is noticeably accompanied with a decrease in the amplitude of the EMG signal [3]. With each vibration repetition, the brain is sharing more and more control over the upcoming contraction with the muscle as the muscle memorizes the activation pattern. With the third vibration application, the brain is potentially not directly involved with the contraction execution. The localised neuroplasticity facilitates control of the last completion over the upcoming contraction, and the evidence could potentially be the mu decreased peak values over the entire sensorimotor cortex.

\section{CONCLUSION}

The results presented in this paper indicate possible beneficial properties associated with focal vibro-tactile muscle preconditioning. The cortical analysis of the mu waves suggests cortical involvement in vibro-tactile facilitation. We postulate that cerebral vibration facilitation is reflected with the increase of the muscle spindle and Golgi tendon organ stretch thresholds, allowing the muscle to stretch and potentially execute greater force with less activated fibres.

There seems to be some indication of neuroplasticity as a result of repetitive vibro-tactile stimulation and cerebral learning process. However, as the presented results are based on a single case study, the analysis of a greater sample is needed in order to investigate the assumptions made. Although all efforts were made to distract the participant from the cues, it is unclear whether visual and audio cues might have been passed to the subject during the inter-connecting neurons at level 1 and within the spinal cord. The use of vibro-tactile stimulation could potentially be integrated with robotic-aided therapy in order to pre-condition muscle response and enhance functional recovery.

\section{ACKNOWLEDGMENT}

The authors wish to thank Middlesex University and Aspire Create for the supporting and funding this study. Special thanks to Dr Tom Carlson for his advice on the EEG data analysis.

\section{REFERENCES}

[1] A. O. Andrade, A. A. Pereira, S. Walter, R. Almeida, R. Loureiro, D. Compagna, and P. J. Kyberd, "Bridging the gap between robotic technology and health care," Biomedical Signal Processing and Control, vol. 10, pp. 65-78, Mar. 2014.

[2] P. Cordo, H. Lutsep, L. Cordo, W. G. Wright, T. Cacciatore, and R. Skoss, "Assisted Movement With Enhanced Sensation (AMES): Coupling Motor and Sensory to Remediate Motor Deficits in Chronic Stroke Patients," Neurorehabilitation and Neural Repair, vol. 23, no. 1, pp. 67-77, Sep. 2008.

[3] T. Jevtic, A. Zivanovic, and R. C. V. Loureiro, "Focal vibrotactile stimulation as a pre-conditioner to enhance muscle performance in robot-mediated neurorehabilitation," IEEE International Conference on Rehabilitation Robotics (ICORR), 2015, pp. 696-701.

[4] M. Sadeghi and B. Sawatzky, "Effects of Vibration on Spasticity in Individuals with Spinal Cord Injury," American Journal of Phys Medicine\& Rehab, vol. 93/11, pp. 995-1007, Nov2014.

[5] M. Mischi, M. Cardinale, "The Effects of a 28-Hz Vibration on Arm Muscle Activity during Isometric Exercise," Medicine \& Science in Sports\&Exercise, vol. 41/3, pp. 645-653, Mar. 2009.

[6] P. Caliandro, C. Celletti, L. Padua, I. Minciotti, G. Russo, G. Granata, G. La Torre, E. Granieri, and F. Camerota, "Focal Muscle Vibration in the Treatment of Upper Limb Spasticity: A Pilot Randomized Controlled Trial in Patients With Chronic Stroke," YAPMR, vol. 93, no. 9, pp. 1656-1661, Sep. 2012.

[7] N. Murillo, J. Valls-Sole, J. Vidal, E. Opisso, J. Medina, and H. Kumru, "Focal vibration in neurorehabilitation.," Eur J Phys Rehabil Med, vol. 50, no. 2, pp. 231-242, Apr. 2014.

[8] M. Etoom and A. Marchetti, "effect of a focal muscle vibration above triceps brachii muscle on upper limb spasticity in a patient with a chronic spinal cord injury: a case report," IJPR, vol. 3, no. 4, pp. 1171-1174, Aug. 2015.

[9] N. Murillo, H. Kumru, J. Vidal-Samso, J. Benito, J. Medina, X. Navarro, and J. Valls-Sole, "Decrease of spasticity with muscle vibration in patients with spinal cord injury," Clinical Neurophysiology, vol. 122, no. 6, pp. 1183-1189, Jun. 2011. J. A. Pineda, Mirror Neuron Systems. Springer Science \& Business Media, 2009.

T. Mima, J. Steger, A. E. Schulman, C. Gerloff, and M. Hallett, "Electroencephalographic measurement of motor cortex control of muscle activity in humans.," Clinical Neurophysiology, vol. 111, no. 2, pp. 326-337, Feb. 2000.

[12] T. W. McDowell, S. F. Wiker, R. G. Dong, and D. E. Welcome, "Effects of vibration on grip and push force-recall performance," International Journal of Industrial Ergonomics, vol. 37, no. 3, pp. 257-266, Mar. 2007.

[13] J. V. Lynskey, A. Belanger, and R. Jung, "Activity-dependent plasticity in spinal cord injury," Journal of reh. research, 2008. T. Kasai, M. Kawanishi, S. Vahagi, "The Effects of Wrist Muscle Vibration on Human Voluntary Elbow Flexion-Extension Movements," Exp Brain Res, vol. 90, no. 1, pp. 217-220, Jul. 1992. 